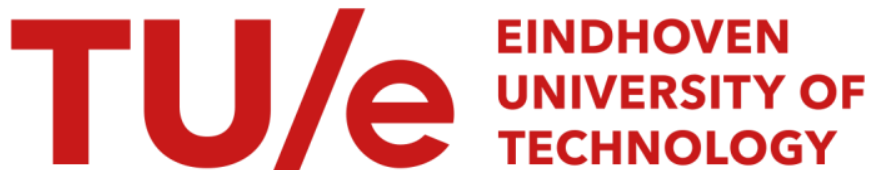

\section{Morphology of symmetric block copolymer in a cylindrical pore}

\section{Citation for published version (APA):}

Sevink, G. J. A., Zvelindovsky, A. V., Fraaije, J. G. E. M., \& Huinink, H. P. (2001). Morphology of symmetric block copolymer in a cylindrical pore. Journal of Chemical Physics, 115(17), 8226-8230.

https://doi.org/10.1063/1.1403437

DOI:

10.1063/1.1403437

Document status and date:

Published: 01/01/2001

\section{Document Version:}

Publisher's PDF, also known as Version of Record (includes final page, issue and volume numbers)

\section{Please check the document version of this publication:}

- A submitted manuscript is the version of the article upon submission and before peer-review. There can be important differences between the submitted version and the official published version of record. People interested in the research are advised to contact the author for the final version of the publication, or visit the $\mathrm{DOI}$ to the publisher's website.

- The final author version and the galley proof are versions of the publication after peer review.

- The final published version features the final layout of the paper including the volume, issue and page numbers.

Link to publication

\section{General rights}

Copyright and moral rights for the publications made accessible in the public portal are retained by the authors and/or other copyright owners and it is a condition of accessing publications that users recognise and abide by the legal requirements associated with these rights.

- Users may download and print one copy of any publication from the public portal for the purpose of private study or research.

- You may not further distribute the material or use it for any profit-making activity or commercial gain

- You may freely distribute the URL identifying the publication in the public portal.

If the publication is distributed under the terms of Article 25fa of the Dutch Copyright Act, indicated by the "Taverne" license above, please follow below link for the End User Agreement:

www.tue.nl/taverne

Take down policy

If you believe that this document breaches copyright please contact us at:

openaccess@tue.nl

providing details and we will investigate your claim. 


\title{
Morphology of symmetric block copolymer in a cylindrical pore
}

\author{
G. J. A. Sevink, A. V. Zvelindovsky, and J. G. E. M. Fraaije \\ Leiden Institute of Chemistry, P.O. Box 9502, 2300 RA Leiden, The Netherlands \\ H. P. Huinink \\ Department of Applied Physics, Eindhoven University of Technology, P.O. Box 513, 5600 MB, Eindhoven, \\ The Netherlands
}

(Received 20 June 2001; accepted 25 July 2001)

\begin{abstract}
The influence of confinement on morphology formation in copolymer systems is an important area of interest in theoretical research. We apply dynamic density functional theory to investigate the effect of pores on the morphology formation in a symmetric diblock copolymer system. The pore is represented by a perfect cylindrical tube. Porous systems are important in biology and are gaining interest for applications in nanotechnology. We show that for the pore sizes under investigation two equilibrium morphologies are possible depending on the surface interaction: a perpendicular or slab morphology and a parallel or multiwall tube morphology. The latter is referred to in the article as dartboard morphology. In the dynamic pathway towards this morphology an intermediate metastable helical phase is found. An important observation is that, for a wide range of pore radii and variations of polymer chain length, no mixed parallel/perpendicular morphologies were found: All observed morphologies are insensitive to the pore diameter. (C) 2001 American Institute of Physics. [DOI: $10.1063 / 1.1403437]$
\end{abstract}

Polymeric materials, such as block copolymers, show a surprisingly rich phase behavior in the mesoscopic domain. Although the building blocks are simple, morphologies range from simple lamellar and hexagonal to complex bicontinuous and cubic phases and even mixed phases. The morphologies are inherent to the topologies of the "building block"; for instance, the ratio of the blocks in the multiblock copolymer. The equilibrium morphologies may in some cases be calculated theoretically, ${ }^{1}$ but in many cases morphologies in experiments are defect-rich and are frozen in at quasiequilibrium states (local minima of the free energy). As a consequence, morphologies resulting from experiments depend heavily on the dynamic pathways. The emphasis in comparing theoretical and experimental results should therefore be on a dynamic theory that includes modulation mechanisms, such as externally applied fields (shear, ${ }^{2}$ electric fields) and confinement effects.

The understanding of the phase behavior of block copolymers in confined systems such as thin films is subject of many theoretical [for instance, by using self-consistent field (SCF) theory $\left.{ }^{3}\right]$ and experimental studies (e.g., Ref. 4). Matsen's theoretical work for symmetric diblocks confined in thin films has been complemented recently by SCF simulations for asymmetric diblocks ${ }^{5}$ that form cylinders in bulk. It was shown that, just as in the case of symmetric diblock copolymer films, parallel layers of cylinders are most frequent. In the case of frustration (film thicknesses that are incompatible with the periodicity of the structure) orthogonal structures are formed. An interesting finding is that the morphologies are not restricted to the bulk morphology: Depending on the confinement and surface interactions we find lamellar and catenoid morphologies.

The experience with these simulations leads to a very straightforward and relevant question: What happens if one frustrates the formation of the most common (lamellar or cylindrical) phases? Does one also observe alternating orientations of the bulk morphologies in this case?

The most reasonable geometry to be considered is a pore. In nature, fluids (water, oil) are often in contact with porous solids. Examples can be found in porous materials in plants (water) and porous inorganic material under the ground (oil and gas). Nanopores are also used in some industrial processes for filtration. An appealing new application is the use of microtubes for application in nanoscopic devices. These microtubes are the result of phase separation in block copolymer systems with hexagonal equilibrium structures, where the necessary well-ordered arrays in the nanoscopic domain in block copolymer systems can be obtained by shearing ${ }^{6,7}$ or by the application of an orienting electric field. ${ }^{8}$ The idea is to replace the tube component, after the equilibration and reorientation, by conducting material while keeping the matrix material inert. Conducting diblock copolymer melts could be used to get structures within the microtubes and well-defined and stable structures when the matrix material is eventually removed. The phase separation process within the tubes is therefore essential and is known to depend on the interaction with the matrix material. A dartboard structure, with conducting-isolating repetition of layers, is most desirable and stable. Validation of simulation results for nanostructures by experimental observations is an important issue. For general porous geometries, an extensive overview of experimental and simulation results is given in Ref. 9. The systems discussed in this review article are simple fluids and binary mixtures, confined in simple (homogeneous) and complex (heterogeneous) pore geometries. The heterogeneity manifests itself in factors such as interpore correlation effects, surface chemical heterogeneity, variations and roughness in pore geometry, and networking of pores. Often these pores are connected to bulk reservoirs of the pore filling materials, implying special boundary 
conditions. In our case, the experimental reference materials are well-tailored, very regular packed cylindrical domains. We consider phase separation of an almost incompressible melt of block copolymers inside one of these cylinders with periodic boundary conditions at the open sides. Phase separation of a block copolymer system implicitly sets the length scales for the microdomains and is in this sense much different from phase separation in binary liquids. We may conclude that this setup, although being rather specific, gives rise to a new generation of experiments.

Quite recently, a Monte Carlo simulation technique was adapted for this kind of geometry. ${ }^{10}$ As in our simulations, a symmetric diblock copolymer system in a cylindrical pore is considered. Although the three-dimensional (3D) results of the two methods overlap, the wider range of the parameter space considered here provides a better insight in the effect of boundary interactions on the morphologies and also allows to analyze the kinetics of the phase separation process. We will compare the results of the two methods in more detail in the discussion of the simulation results. In our case the diblock copolymers are modeled by a $A_{i} B_{i}$ Gaussian chain for different values of the chain length $i$. Since these diblocks form lamellae in bulk and perpendicular lamellae in neutral slits (see the discussion in Ref. 5), we expect frustration of this morphology in the middle of the pore. As in the numerical experiments for confined thin films, ${ }^{5}$ the important parameters are the degree of confinement, i.e., the pore radius $R$, and the effective surface interactions $\xi_{A B}=\left(\epsilon_{A M}^{0}\right.$ $\left.-\epsilon_{B M}^{0}\right) / \nu k T$, where $\epsilon_{I M}^{0}$ denotes the scalar interaction ${ }^{11}$ of bead $I$ with the pore boundary. Since the diblock is symmetric, we make use of reciprocity and restrict ourselves to values of $\xi \geqslant 0$. We use simulations with SCF theory ${ }^{12}$ to construct a morphology diagram as a function of $R$ and $\xi$. As it turns out, for the radii under consideration only two morphologies are possible: a slab and a dartboard morphology. Finally we will consider the dynamic pathway towards the slab morphology in great detail.

The time evolution of the density field $\rho_{I}(\mathbf{r})$ is described by a time-dependent Landau-Ginzburg-type equation with a stochastic term $\eta_{I}$ (Refs. 13 and 14):

$$
\dot{\rho}_{I}=M_{I} \nabla \cdot \rho_{I} \nabla \frac{\delta F}{\delta \rho_{I}}+\eta_{I},
$$

where $M_{I}$ is a mobility parameter and $I$ is a component index. The free energy for a collection of $n$ Gaussian chains is given by ${ }^{11,12}$

$$
\begin{aligned}
F= & -k T \ln \frac{\Phi^{n}}{n !}-\sum_{I} \int_{V} U_{I}(r) \rho_{I}(r) d r \\
& +\frac{1}{2} \sum_{I, J} \int_{V} \int_{V} \epsilon_{I J}\left(r-r^{\prime}\right) \rho_{I}(r) \rho_{J}\left(r^{\prime}\right) d r d r^{\prime} \\
& +\frac{1}{2} \sum_{I} \int_{V} \epsilon_{I M}\left(r-r^{\prime}\right) \rho_{I}(r) \rho_{M}\left(r^{\prime}\right) d r d r^{\prime} \\
& +\frac{1}{2} \kappa_{H} \nu^{2} \int_{V}\left(\sum_{I} \rho_{I}(r)-\sum_{I} \bar{\rho}_{I}\right)^{2} d r .
\end{aligned}
$$

The first two terms account for the entropy of a system of $n$

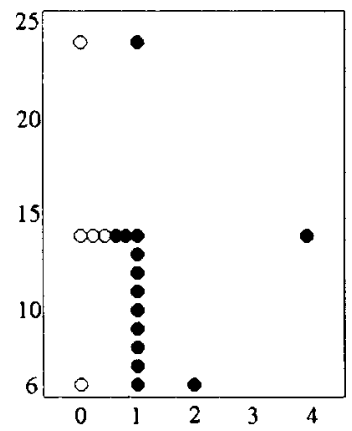

FIG. 1. Phase diagram for the $A_{8} B_{8}$ diblock morphologies as a function of pore diameter $R$ (vertical axis) and effective interaction $\xi$ (horizontal axis). Circles denote the points in the phase diagram that have been simulated. Open circles represent slab geometries; closed circles dartboard geometries.

ideal chains subject to an external potential field $U_{I}$ (with $\Phi$ the partition function for ideal chains), the third and fourth terms for the cohesive bead-bead and bead-wall interactions, and the final term for the excluded volume interactions, with $\nu$ the bead volume and $\kappa_{H}$ the Helfand compressibility parameter. Confinement is accounted for in the free energy by the fourth term [and $\theta_{M}(r)=\nu \rho_{M}(r)$ is nonzero $(=1)$ only in the solid objects] and the boundary condition for the dynamic equations $n \cdot \nabla \mu_{I}=0$, with $n$ the normal pointing into the solid object. ${ }^{12}$ In contrast to traditional schemes of polymer phase separation dynamics where a Landau Hamiltonian is used with vertex functions calculated following the random phase approximation (see, e.g., Ref. 6), we numerically calculate the "exact" free energy $F$ of polymer system consisting of Gaussian chains in mean field environment using path integral formalism. ${ }^{13,14}$ Our approach uses essentially the same free energy functional as in SCF calculations of equilibrium block copolymer morphologies by Matsen and Schick ${ }^{15}$ and Drolet and Fredrickson, ${ }^{16}$ but complements the static SCF calculations by providing a dynamical picture of the system, which is crucial for the evolution of system in an external fields. ${ }^{17-19}$

We have performed simulations for a symmetric diblock copolymer system. The chain architecture was chosen as $A_{8} B_{8}$. We have used this system in many earlier simulations and therefore the relevant parameters can be found in Ref. 12 .

The equilibrium structure for this diblock system is lamellar. Figure 1 shows the phase diagram for the diblock system. Due to the symmetry only positive values of $\xi$ are considered. In the dartboard part, equilibrium morphologies were reached at a much earlier stage than the slab part $(y$ $=2000$ to $y=7000$, with $y=t / \Delta t$ ). The value of $\Delta t$ is chosen equal for all simulations, such that $y$ can be directly compared. Although the number of calculated points is relatively small, the relative position of the phase boundaries is quite obvious.

We first study the influence of confinement by considering different radii $R$ for $\xi=0$. One should note that $\xi=0$ represents all phase points where the block-surface interactions is equal for both species (neutral surface). The results of this exercise are shown in Fig. 2. We note that the final morphology for all different radii is a slab morphology. The 


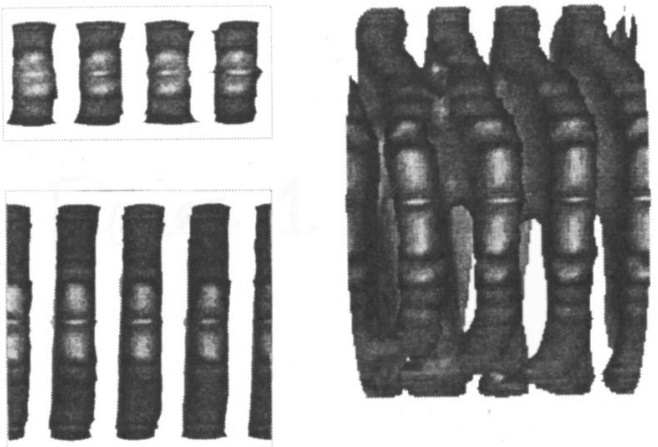

FIG. 2. Morphologies at dimensionless time $y=10000$ for the $B$ component of the diblock copolymer melt in a cylinder (side view) of radius of 8,14 , and 24 grid points and $\xi=0$. Orthogonal projections are shown of isosurfaces $\left(\nu \rho_{B}=0.4\right.$ with $\nu$ the bead volume). The length is in all cases equal to 32 grid points.

difference between the three results is in the kinetics: apparently the system needs more time to equilibrate for larger radii $R$.

Looking at the time behavior (only shown for $R=14$ in Fig. 3) shows us that the equilibrium structure is reached at $y=6000$ for $R=6$ and at $y=8000$ for $R=14$. For the largest radius under consideration $R=24$, the slab morphology is not yet fully developed at $y=10000$, showing that the kinetics of slab morphology formation depends greatly on the radius. However, it is obvious that the slab morphology is the equilibrium morphology for these simulations. Challenging the morphology of $R=14$ with externally applied shear ${ }^{7,20}$ (results not shown here) leads to a breaking up of the slabs. However, upon cessation of shear, the slab morphology is finally completely recovered.

In Ref. 10 the case of neutral walls is only considered in two dimensions. With preferential wetting of one of the blocks the dartboard structure is obtained both in two and three dimensions. For the neutral case it is shown in two
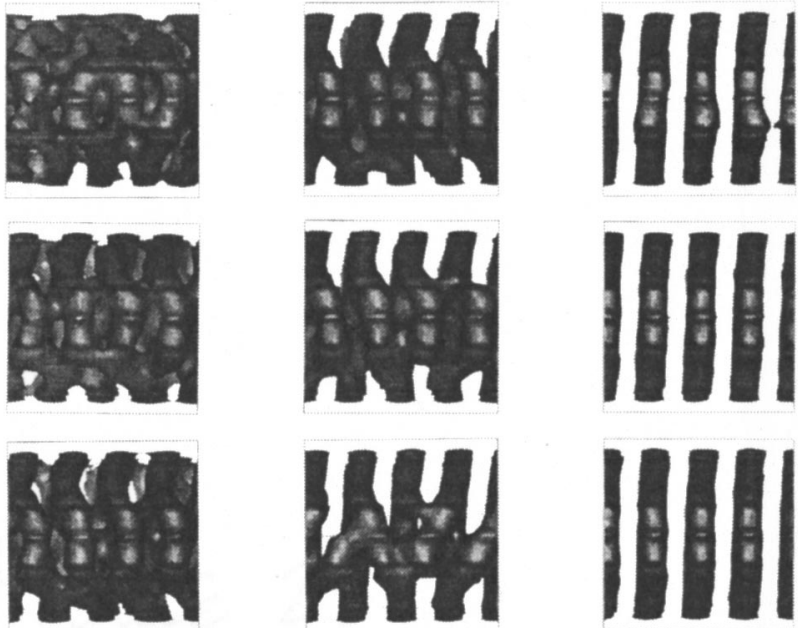

FIG. 3. Time development (upper left to lower right) of the diblock copolymer morphology in a cylinder of radius $R=14$ grid points. Orthogonal projections are shown of isosurfaces $\left(\nu \rho_{B}=0.4\right)$ and for every subsequent 1000 time steps. One can clearly see that the structure "switches" in directional orientation for going to a stable slab phase. dimensions that, when the dartboard structure (strip phase in the notation of that article) is frustrated, a "disordered" state is obtained. This disordered state is a lamellar state, in which the global orientation is random, and the lamellae are locally oriented perpendicular to the wall. The mechanism behind the formation is stated as "currently underway." From our simulations we conclude that this "disordered" structure is a 2D artifact: there is not enough freedom to form a perfect structure. In three dimensions, due to the extra degree of freedom, instead the slab morphology is formed.

If we introduce a moderate surface interaction $(\xi=1)$ the time behavior changes drastically. Dartboard morphologies are formed at a fast rate, indifferent to the pore radii. In Fig. 4 , we show front views of equilibrium morphologies for the radii under consideration for both blocks. The corresponding time stages are $y=4000(R=6), y=5000(R=14)$, and $y$ $=2000(R=24)$. The perfect morphologies are formed at roughly the same time stages $y \approx 2000$, and become more profound at later time stages. From this, we may conclude that the kinetics of the formation is independent of the pore radius. One can clearly observe the dartboard structures (concentric cylinders). Since the surface is $A$-repulsive, the $B$ block is present at the surface. Again we stress the fact that only the difference in interaction values (the effective interaction $\xi$ ) counts. Increasing the effective surface interaction leads to similar dartboard structures, although the layer thickness may vary and the middle layers are somewhat compressed.

In order to find regions of frustration of this morphology, we performed simulations for different intermediate pore radii (and $\xi=1$ ): $R=7-13$. We observed dartboard morphologies with different alternation of species rings (counted from the center): $B-A-B(R=7-9), A-B-A-B(R=10-12)$, and $B-A-B-A-B(R=13)$. All these morphologies were well developed at $y=2000$ and as expected, the $B$ block is always at the surface. These results are all directly comparable to the multibarrel-layer results of $\mathrm{He}$ (Ref. 10) (Fig. 5 in that article).

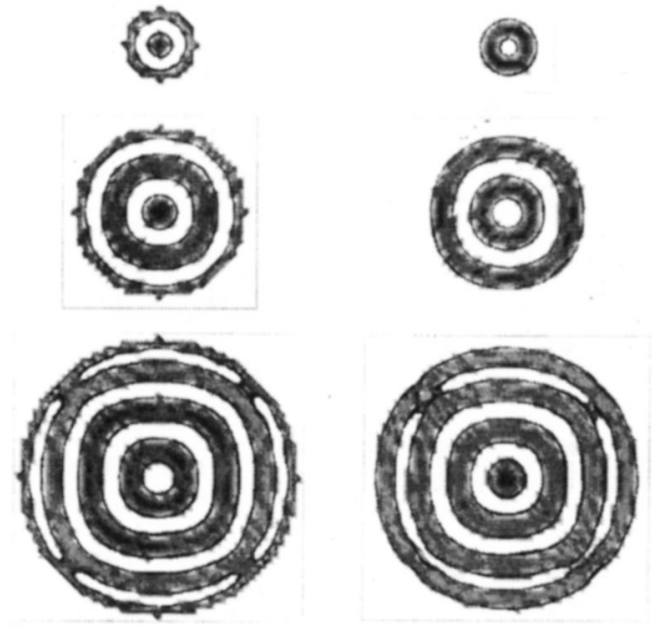

FIG. 4. Parallel projection of isosurface $\left(\nu \rho_{I}=0.4, I=A\right.$ or $B$, front view) and orthoslice of final structures for different radii: 8,14 , and 24 gridpoints. Left, $B$ blocks; right, $A$ blocks. 

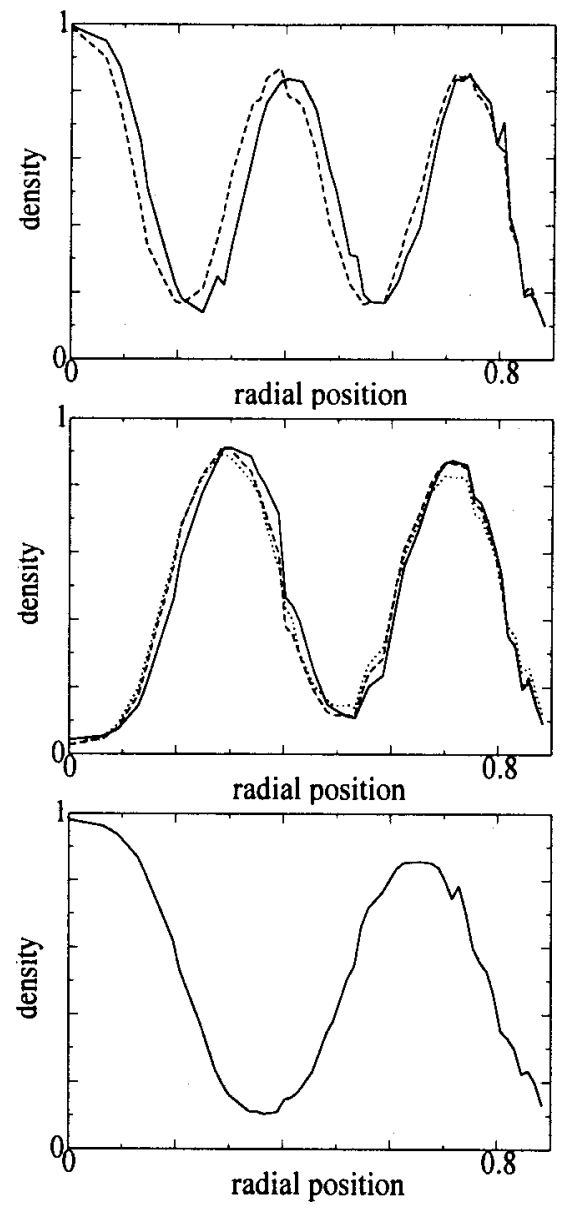

FIG. 5. Angle-averaged density profiles ( $\left.\nu \rho_{A}\right)$ for different chains $A_{i} B_{i}$ as a function of radial position (here $r / R$ ). Top: $i=3$ (solid) and $i=4$ (dashed). Middle: $i=5$ (solid), $i=6$ (dashed), and $i=8$ (dotted). Bottom: $i=16$.

In order to understand the influence of the chain length, and the degree of compression and stretching of the chains, we have also varied the chain length of the diblock. We carried out simulations for $\left(A_{i} B_{i}\right.$ for $i=3-6$ and 16) for fixed $R=13$ and a $\xi$ such that $\xi N=16$ (with $N=2 i$ the number of beads) is fixed. In all cases this leads to fast formation of a perfect dartboard morphology, with alternating $A$ - and $B$-rich layers as $A-B-A-B-A-B(i=3$ and 4$), B-A-B-A-B(i$ $=5$ and 6 ), and $A-B-A-B(i=16)$ (again counted from the center of the pore). In the top part of Fig. 5, we show angleaveraged density profiles $\nu \rho_{A}(r)$ for the $A$ block, where $r$ is the radial position. We observe great similarity between profiles of similar repetition: $i=3$ and 4 (top); $i=5,6$, and 8 (middle). We also compared the profiles when the radial position is scaled by $\sqrt{N} \sim R_{g}$, where $R_{g}$ is the root mean squared (rms) end-to-end length of an unperturbed Gaussian chain, shown in Fig. 6. Here, the compared profiles share the center species: $i=3,4$, and 16 (upper) and $i=5,6$, and 8 (lower). It can be seen from these figures that the profile for the center regions is relatively similar for all chain sizes with similar center species. At the center, the compression is large, as can be seen from the total density (which is higher than unity). Further away from the center region the chains are relatively less compressed, which can be seen from the fact that the behavior is rather independent from the chain length.
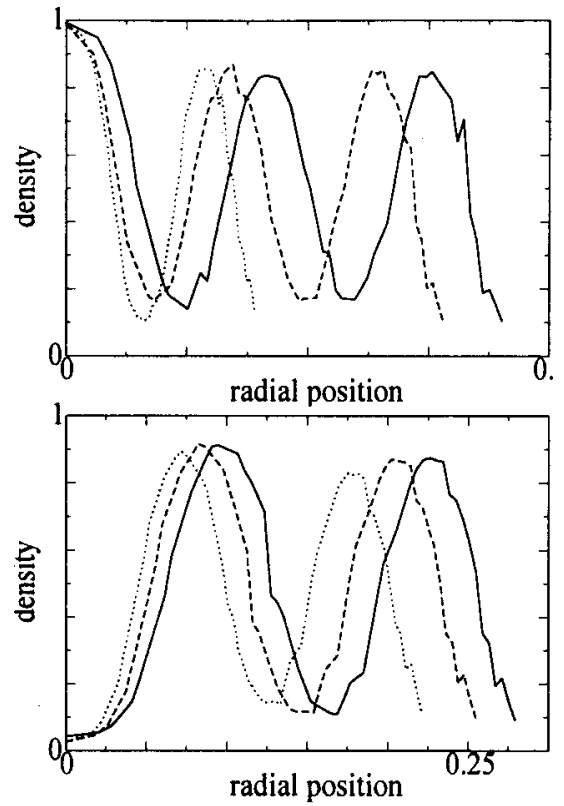

FIG. 6. Angle-averaged density profiles $\left(\nu \rho_{A}\right)$ for different chain lengths as a function of radial position. The horizontal axis of Fig. 5 is scaled with respect to the different $R_{g}$ [the radial position denotes $r /\left(R R_{g}\right)$ ]. Top: $i$ $=3$ (solid), $i=4$ (dashed), and $i=16$ (dotted). Bottom: $i=5$ (solid), $i=6$ (dashed), and $i=8$ (dotted).

Apparently, the freedom of the system to increase the compression in the center region and adjust the layer sequence is such that little frustration is present close to the pore boundary.

In order to determine the phase boundary, we simulated $A_{8} B_{8}$ for a radius $R=14$ and values of $\xi=0+0.2 I$ for $I$ $\in\{1,2,3,4\}$. We found that for this radius the phase boundary should be between $\xi=0.4$ (slab phase) and $\xi=0.6$ (dartboard phase) (see also Fig. 1). The position of the phase boundary for this radius cannot simply be extrapolated to other radii, due to the dependence of the dynamics to the radius of the pore. However, since we are not interested in exact determination of the phase boundaries for all $R$ (they depend also on the sizes of the blocks for instance) we do not consider other radii.

Finally, we consider the dynamic behavior for one phase point in the slab morphology part in more detail. The system considered is again the $A_{8} B_{8}$ diblock copolymer melt. The particular confinement and interaction parameters are $\xi=0$ and $R=14$. The time behavior discussed here is typical for the development of a slab morphology in three dimensions. In Fig. 3 we show the time development for this phase point. From upper left to lower right we show the morphologies $\left[\nu \rho_{A}(r)=0.4\right]$ for $y=1000 k, k=1, \ldots, 10$. We observe that the phase separation process is fairly slow: the initial messy morphology at $y=1000$ turns slowly into a more defined morphology at $y=4000$. This phase is a very interesting one: it resembles a helical phase. It is built up of twisted slabs with winding interconnections just as in a helical structure, apart from the fact that the axis of winding is not precisely in the middle of the pore but fluctuates. This phase is not a stable one but an intermediate result. Further development does not immediately lead to a removal of the defects but 
rather a reorientation of the convections and a straightening of the slabs $(y=5000-6000)$. Only at $y=7000$ a slab morphology evolves to full extend. However, even at $y$ $=10000$, the slabs do not completely aline perpendicular to the pores boundary but experience a slight tilt.

Concluding, we observe two phases in a symmetric diblock copolymer melt depending on the energetic wallpolymer interactions: a slab morphology for neutral or almost neutral interactions, and a dartboard morphology for stronger preferential interactions. These phases can be characterized as perpendicular lamellae (slab morphology) and parallel lamellae (dartboard morphology). In this sense, both phases that are found for systems confined between parallel plates $^{3}$ are found here as well. Other morphologies have not been found with a wide variation of the radius in the dartboard morphology part of the morphology diagram. Also variation of the block lengths did not lead to other morphologies, and the system seems to have enough freedom to not experience any mixed orthogonal/parallel oriented morphologies. The formation of the observed morphologies differs in kinetics. The slab morphology has slow kinetics, depending on the pore size, and experiences a helical-like structure as an intermediate but long-living state. The kinetics of the dartboard phase formation seems to be independent of the pore radius, for the range of radii under consideration.

The authors acknowledge support of NCF (Stichting Nationale Computer Faciliteiten). We thank T. Russell for pointing out the application of pores in nanotechnology.

${ }^{1}$ F. S. Bates and G. H. Fredrickson, Phys. Today 52(2), 32 (1999).
${ }^{2}$ A. V. Zvelindovsky and G. J. A. Sevink, and J. G. E. M. Fraaije, Phys. Rev. E 62, R3063 (2000).

${ }^{3}$ M. W. Matsen, J. Chem. Phys. 106, 7781 (1997).

${ }^{4}$ G. J. Kellogg, D. G. Walton, A. M. Mayes, P. Lambooy, T. P. Russell, P. D. Callager, and S. K. Satija, Phys. Rev. Lett. 14, 2503 (1996).

${ }^{5}$ H. P. Huinink, J. C. M. Brokken-Zijp, M. A. van Dijk, and G. J. A. Sevink, J. Chem. Phys. 112, 2452 (2000).

${ }^{6}$ I. W. Hamley, The Physics of Block Copolymers (Oxford University Press, Oxford, 1998)

${ }^{7}$ A. V. M. Zvelindovsky, B. A. C. van Vlimmeren, G. J. A. Sevink, N. M. Maurits, and J. G. E. M. Fraaije, J. Chem. Phys. 109, 8751 (1998).

${ }^{8}$ T. Thurn-Albrecht, J. deRouchey, T. P. Russell, and H. M. Jaeger, Macromolecules 33, 3250 (2000).

${ }^{9}$ L. D. Gelb, K. E. Gubbins, R. Radhakrishnan, and M. SliwinskaBartkowiak, Rep. Prog. Phys. 62, 1573 (1999).

${ }^{10}$ X. He, M. Song, H. Liang, and C. Pan, J. Chem. Phys. 114, 10510 (2001).

${ }^{11}$ J. G. E. M. Fraaije, B. A. C. van Vlimmeren, N. M. Maurits, M. Postma, O. A. Evers, C. Hoffmann, P. Altevogt, and G. Goldbeck-Wood, J. Chem. Phys. 106, 4260 (1996).

${ }^{12}$ G. J. A. Sevink, A. V. Zvelindovsky, B. A. C. van Vlimmeren, N. M. Maurits, and J. G. E. M. Fraaije, J. Chem. Phys. 110, 2250 (1999).

${ }^{13}$ J. G. E. M. Fraaije, J. Chem. Phys. 99, 9202 (1993); 100, 6984 (1994); J. G. E. M. Fraaije, B. A. C. van Vlimmeren, N. M. Maurits, M. Postma, O. A. Evers, C. Hoffmann, P. Altevogt, and G. Goldbeck-Wood, ibid. 106, 4260 (1997).

${ }^{14}$ B. A. C. van Vlimmeren, N. M. Maurits, A. V. Zvelindovsky, G. J. A. Sevink, and J. G. E. M. Fraaije, Macromolecules 32, 646 (1999).

${ }^{15}$ M. W. Matsen and M. Schick, Phys. Rev. Lett. 72, 2660 (1994).

${ }^{16}$ F. Drolet and G. H. Fredrickson, Phys. Rev. Lett. 83, 4317 (1999).

${ }^{17}$ H. Kodama and M. Doi, Macromolecules 29, 2652 (1996).

${ }^{18}$ A. Onuki, J. Phys.: Condens. Matter 9, 6119 (1997).

${ }^{19}$ T. Kawakatsu, Phys. Rev. E 56, 3240 (1997); 57, 6214 (1998).

${ }^{20}$ A. V. Zvelindovsky, G. J. A. Sevink, B. A. C. van Vlimmeren, N. M. Maurits, and J. G. E. M. Fraaije, Phys. Rev. E 57, R4879 (1998); Prog. Colloid Polym. Sci. 110, 251 (1998). 